\begin{tabular}{|c|c|c|c|c|c|}
\hline & $\begin{array}{l}R_{0} \\
\AA\end{array}$ & $\begin{array}{c}P \\
\mathrm{kcal} / \mathrm{mol} \cdot \AA\end{array}$ & $\stackrel{Q}{\mathrm{kcal} / \mathrm{mol} \cdot \AA^{2}}$ & $\stackrel{A}{\mathrm{kcal} / \mathrm{mol} \cdot \AA^{6}}$ & $\begin{array}{c}B \\
\mathrm{kcal} / \mathrm{mol} \cdot \AA^{8}\end{array}$ \\
\hline \multirow[t]{2}{*}{$\begin{array}{c}\text { Alkohol } \\
\text { Eis }\end{array}$} & $\begin{array}{l}2,69 \\
2,76\end{array}$ & $\begin{array}{l}55,65 \\
54,95\end{array}$ & $\begin{array}{l}-140,5 \\
-136,5\end{array}$ & $\begin{array}{l}547 \\
905\end{array}$ & $\begin{array}{l}4,40 \cdot 10^{7} \\
4,50 \cdot 10^{7}\end{array}$ \\
\hline & $\begin{array}{c}E_{\mathrm{HO}} \\
\mathrm{kcal} / \mathrm{mol}\end{array}$ & $\begin{array}{c}U \text { Disp. } \\
\mathrm{kcal} / \mathrm{mol}\end{array}$ & $\begin{array}{c}U_{\text {Abst. }} \\
\mathrm{kcal} / \mathrm{mol}\end{array}$ & $\begin{array}{l}\text { Brückenl } \\
\text { (theor.) } \\
\text { kcal/mol }\end{array}$ & $\begin{array}{c}\text { gsenergie } E \\
\text { (exp.) } \\
\text { kcal/mol }\end{array}$ \\
\hline $\begin{array}{l}\text { Alkohol } \\
\text { Eis }\end{array}$ & $\begin{array}{r}14,34 \\
-\quad 9,93\end{array}$ & $\begin{array}{l}-1,44 \\
-2,05\end{array}$ & $\begin{array}{r}10,99 \\
7,56\end{array}$ & $\begin{array}{l}4,8 \\
4,4\end{array}$ & $\begin{array}{l}6 \\
5\end{array}$ \\
\hline
\end{tabular}

Tab. 7. Anteile der Wasserstoffbrückenbindungsenergie.

(28) und (30) so dar:

$$
\begin{aligned}
U(R) & =U\left(R_{1}\right)+P\left(R-R_{1}\right) \\
& +Q\left(R-R_{1}\right)^{2}-\frac{A}{R^{6}}+B R^{8} e^{-8,6 \cdot R}
\end{aligned}
$$

$R_{1}$ ist ein dem Gleichgewichtsabstand $R_{0}$ nahe liegender Abstandswert aus Tab. $6\left(R_{1}=2,7 \AA\right) . B$ bestimmt sich einfach aus der Gleichgewichtsbedingung für das Potential (31) :

$$
(\mathrm{d} U / \mathrm{d} R)_{R=R \mathbf{0}}=0 .
$$

Die Ergebnisse sind in Tab. 7 zusammengestellt.

Wenn an dem Atom $\mathrm{O}_{\mathrm{I}}$ ebenfalls eine Wasserstoffbrücke angreift, verstärkt sich die Energie der $\ldots \mathrm{O}_{\mathrm{I}}-\mathrm{H} \ldots \mathrm{O}_{\mathrm{II}}-$ Wechselwirkung. Dieser Effekt ist in den theoretischen Daten nicht berücksichtigt, weshalb die berechneten Werte der Brückenbindungs- energie etwas zu klein sein müssen. Aus den Sublimationsenergien für Methylalkohol und Eis $(9,8$ bzw. 11,8 kcal $/ \mathrm{mol}$ ) lassen sich, nach Abzug überschüssiger Anteile (Dispersionsenergie der $\mathrm{CH}_{3}$ Gruppen und Polarisationseffekte bei Methylalkohol, Einfluß der nicht nächsten Nachbarn bei Eis), experimentelle Werte der ... O-H ... O-H ....Wechselwirkungsenergie schätzen, die den berechneten etwa entsprechen. Die Übereinstimmung ist recht befriedigend und man wird annehmen dürfen, daß die Anteile der Brückenbindungsenergie ungefähr richtig angegeben sind.

Herrn Professor Friedrich Hund danke ich für Förderung und Unterstützung. Mein Dank gilt auch Herrn Professor KARL Wirtz und der Rechengruppe des MaxPlanck-Instituts für Physik, die die Durchführung dieser Arbeit ermöglichten.

\title{
Ringförmiges Elektronengasmodell zur Beschreibung der Zustände der $\pi$-Elektronen in aromatischen Kohlenwasserstoffen
}

\author{
Von H. BAUR * \\ Aus dem Institut für theoretische Physik der Universität Mainz \\ (Z. Naturforschg. 13 a, 1057-1066 [1958]; eingegangen am 29. Juli 1958)
}

\begin{abstract}
Zur Beschreibung der stationären Zustände der $\pi$-Elektronen in aromatischen Kohlenwasserstoffen wird ein ringförmiges Elektronengasmodell eingeführt, das gestattet, in einfacher Weise die Bindungsverhältnisse der $\pi$-Elektronen zu berechnen. Die sog. Sonderenergie der Aromaten ergibt sich im wesentlichen als Coulombsche Wechselwirkungsenergie der $\pi$-Elektronen untereinander.
\end{abstract}

Zur Beschreibung der stationären Zustände der $\pi$-Elektronen in ungesättigten und aromatischen Kohlenwasserstoffen wurde von KuHN und anderen mit Erfolg das Kastentestverfahren verwandt ${ }^{1}$. Insbesondere lassen die Zustände der $\pi$-Elektronen in

* Dissertation, Mainz 1958 (gekürzte Fassung).

1 Eine Einführung in die Problemstellung findet sich in Experientia 9, 2 [1953]. den Acenen sich recht gut mit dem eindimensionalen verzweigten Elektronengasmodell darstellen ${ }^{2}$. Bei der Anwendung dieses Modells auf die $\pi$-Elektronen der Phene erhält man jedoch keinerlei Übereinstimmung mit der Erfahrung. Dies läßt vermuten, daß bei den

\footnotetext{
2 H. H. Perkampus, Z. Naturforschg. 7 a, 9 [1952]; Z. physik. Chem., N. F. 2, 3/4 [1954].
} 
Phenen eine Ausbreitung des einzelnen $\pi$-Elektrons über das ganze Molekül, wie sie mit dem verzweigten Elektronengasmodell angenommen wird, nicht möglich ist.

Es wurde nun versucht, durch Abänderung der Form der dem eindimensionalen Modell zugrundeliegenden Testkästen ein auch für die $\pi$-Elektronen der Phene brauchbares Modell zu finden. Die Form der Testkästen wurde gewonnen durch Vergleich der Kekulé-Strukturen und durch Vergleich der Lage der langwelligsten Absorptionsmaxima gewisser Molekülgruppen. Im folgenden wird die eindimensionale Beschreibung dieses Modells dargelegt.

\section{Eindimensionales, ringförmiges Elektronengas- modell}

Bei den Acenen nehmen wir zunächst an, daß die $\pi$-Elektronen sich ober- und unterhalb der Ebene der C-Atomschwerpunkte längs ringförmiger Streckenzüge der Form

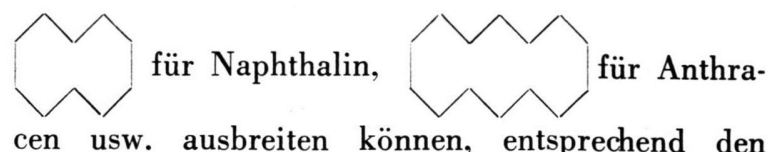
KEKULÉ-Strukturen

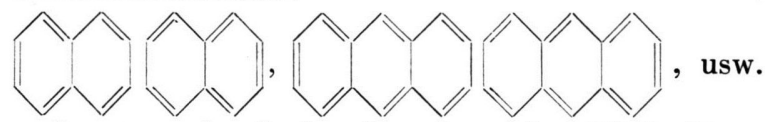

und entsprechend der Zunahme der Wellenlänge des langwelligsten Absorptionsmaximums mit der Annellierung ${ }^{3}$.

Die Wellenlänge des langwelligsten Absorptionsmaximums der Phene wird bei linearer Annellierung eines Benzolringes wie bei den Acenen vergrößert, bei angularer Annellierung ändert sich ihr Wert jedoch kaum ${ }^{3}$. Dies legt nahe, bei Phenen die Beweglichkeit der $\pi$-Elektronen auf ringförmige Streckenzüge zu beschränken, die nur die linear annellierten Teile des Moleküls umfassen; bei Phenanthren etwa auf Streckenzüge, wie sie in den folgenden Bildern durch die ausgezogenen Linien angedeutet werden:
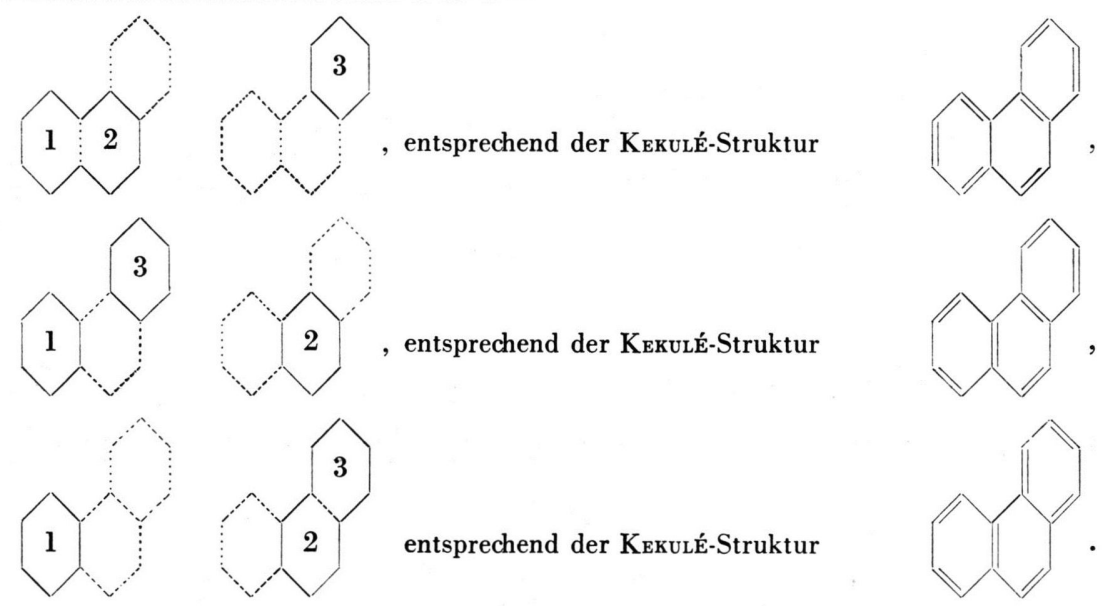

Im Einklang damit steht, daß sich keine Anzeichen für das Vorhandensein der Strukturen<smiles>c1ccc2c(c1)ccc1ccccc12</smiles>

und

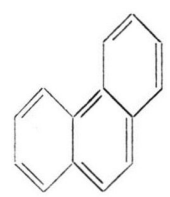

finden ${ }^{4}$.

Eine Beschränkung dieser Art kann man schließ-

3 Die Absorptionsspektren der aromatischen Kohlenwasserstoffe finden sich in einer vergleichenden Ubersicht bei lich für einzelne $\pi$-Elektronen auch bei den Acenen annehmen, entsprechend den KEKULÉ-Strukturen und den kurzwelligeren Absorptionsbanden; bei Naphthalin z. B. auf Streckenzüge der Form

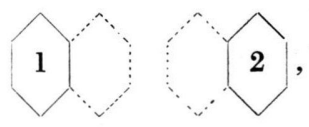

entsprechend der KEKULÉ-Struktur

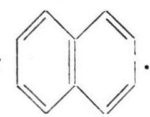

E. Clar, Aromatische Kohlenwasserstoffe, 2. Aufl., Springer-Verlag, Berlin 1952.

4 Vgl. Einleitung und E. Clar, l. c. ${ }^{3}$, S. 81/82. 
Wir nehmen also an, daß die $\pi$-Elektronen eines aromatischen Moleküls sich ober- und unterhalb der Ebene der C-Atomschwerpunkte auf ringförmige Streckenzüge verschiedener Größe verteilen. Diese Streckenzüge wollen wir $\pi$-Ringe nennen. Für die $\pi$-Ringe in den Acenen und Phenen führen wir speziell die Bezeichnungen

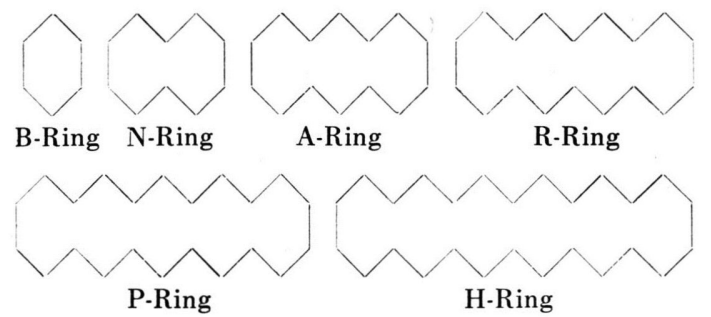

ein. Die Länge des größten $\pi$-Ringes in einem Molekül soll, wie oben begründet, bei den Acenen und Phenen durch die Anzahl der im betreffenden Molekül linear annellierten Benzolringe gegeben sein. Zum Beispiel sollen die $\pi$-Elektronen sowohl in Anthracen als auch in Tetraphen und 8,9-Benztetraphen in ihrer Ausbreitungsmöglichkeit auf B-, $\mathrm{N}$ - und A-Ringe beschränkt sein; insbesondere in Anthracen $1 \begin{array}{lll} & 2 & 3\end{array}$ auf drei B-Ringe $B_{1}, B_{2}, B_{3}$ :
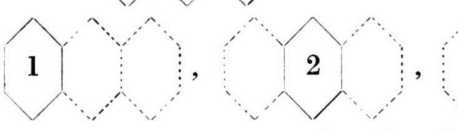
3

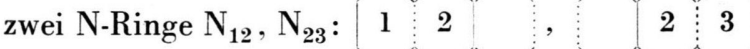
und auf einen A-Ring $\mathrm{A}_{13}: 1: 3$.

Über die Besetzung der einzelnen Ringe mit $\pi$-Elektronen müssen die Symmetrie- und Energieverhältnisse Auskunft geben.

\section{Einfaches Ring-Modell, ohne analytische Beschreibung der Dichtefunktionen ${ }^{5}$ der $\pi$-Elektronen}

\section{Die Einelektronen-Eigenfunktionen}

Auf jedem $\pi$-Ring definieren wir eine Linienkoordinate $x_{\pi}(\pi=\mathrm{B}, \mathrm{N}, \mathrm{A} \ldots)$. Die Länge eines $\pi$-Ringes bezeichnen wir mit $L(\pi)$. Als Längen-

5 Unter der Dichte von Elektronen wird hier und im folgenden die "Dichte“ der Aufenthaltswahrscheinlichkeit von Elektronen verstanden. einheit $l_{0} \triangleq 1$ wählen wir den mittleren Abstand der Schwerpunkte zweier C-Atome in der aromatischen C-C-Bindung mit 50\% Doppelbindungscharakter. $L(\pi)$ betrage stets ein ganzzahliges Vielfaches dieser Einheit, so daß also

$$
L(\mathrm{~B})=6, \quad L(\mathrm{~N})=10, \quad L(\mathrm{~A})=14, \text { usw. wird. }
$$

Nehmen wir eine freie Ausbreitung der $\pi$-Elektronen längs der $\pi$-Ringe an, so ist deren Aufenthaltswahrscheinlichkeit gegeben durch die Funktionen

$$
\left.\begin{array}{rl}
\psi_{0}\left(x_{\pi}\right) & =\text { const }=\left[\frac{1}{L(\pi)}\right]^{1 / 2} ; \\
\psi_{n}{ }^{(1)}\left(x_{\pi}\right) & =\left[\frac{2}{L(\pi)}\right]^{1 / 2} \sin \frac{\varkappa n}{L(\pi)} x_{\pi} ; \\
\psi_{n}{ }^{(2)}\left(x_{\pi}\right) & \left.=\left[\frac{2}{L(\pi)}\right]^{1 / 2} \cos \frac{\varkappa n}{L(\pi)} x_{\pi} \quad \text { (s. Anm. }{ }^{6}\right) ; \\
n & =1,2,3 \ldots
\end{array}\right\}
$$

mit den Energiewerten

$$
\varepsilon_{\mathrm{kin}}=\frac{h^{2}}{8 m l_{0}^{2}}\left[\frac{n}{L(\pi)}\right]^{2} ; \quad n=0,1,2 \ldots
$$

( $m=$ Masse des Elektrons, $h=$ Plancksches Wirkungsquantum).

Durch (2) ist, da eine freie Beweglichkeit der Elektronen auf den $\pi$-Ringen ein konstantes Potential längs der Ringe voraussetzt, bis auf eine additive Konstante, auch die Gesamtenergie $\varepsilon$ der einzelnen $\pi$-Elektronen gegeben.

$\mathrm{Zu}$ jedem Ring existieren also ein nicht entarteter Grundzustand und einfach entartete angeregte $\mathrm{Zu}$ stände. Die Energie des Grundzustandes ist unabhängig von der Länge des Ringes. Die Energie der angeregten Zustände ist, davon wurde schon bei der Begründung des Ringmodells in Abschnitt I Gebrauch gemacht, umgekehrt proportional $\mathrm{zu} L^{2}(\pi)$.

Eine völlig freie Bewegung der $\pi$-Elektronen längs der $\pi$-Ringe dürfen wir jedoch nicht voraussetzen. Vielmehr ist das einzelne Elektron, durch die Wirkung der auf den anderen Ringen lokalisierten Elektronen, längs seines Ringes einer Störung ausgesetzt, die meist nicht die volle Symmetrie des Ringes besitzt und die auch nicht angenähert als konstant betrachtet werden darf. Nur so können wir überhaupt die Beschränkung der Ausbreitungsmöglichkeit der $\pi$-Elektronen auf einzelne Ringe erklären. Andererseits sprechen aber die dielektrischen und diamagnetischen Eigenschaften der aromatischen Kohlenwasserstoffe doch für eine recht große

\footnotetext{
6 Um Verwechslungen mit der Ringbezeichnung zu vermeiden, wurde für die sonst mit $\pi$ bezeichnete Zahl 3,14159 ... $\varkappa$ gesetzt.
} 
Beweglichkeit der $\pi$-Elektronen längs der $\pi$-Ringe. In Anlehnung an (1) und (2) setzen wir daher für die Dichtefunktionen $\psi\left(x_{\pi}\right)$ voraus, daß

(V1) zu jedem $\pi$-Ring ein nicht entarteter Grundzustand $\psi_{0}\left(x_{\pi}\right) \equiv(\pi)$ mit der Energie $\varepsilon(\pi)$ existiert,

(V2) die Grundzustände der einzelnen Ringe untereinander entartet sind bzw. sich energetisch nur wenig unterscheiden: $\varepsilon(\pi)=$ const $=\varepsilon_{0}$ unabhängig von $L(\pi)$,

(V3) zu jedem $\pi$-Ring ein erster angeregter $\mathrm{Zu}$ stand $\psi_{1}\left(x_{\pi}\right) \equiv\left(\pi^{\prime}\right)$ existiert, dessen Energie $\varepsilon\left(\pi^{\prime}\right)=\varepsilon(\pi)+\Delta \varepsilon\left(\pi^{\prime}\right)$ betrage, wobei $\Delta \varepsilon\left(\pi^{\prime}\right)$ umgekehrt proportional zu $L^{2}(\pi)$ sei:

$$
\Delta \varepsilon\left(\pi^{\prime}\right)=\gamma / L^{2}(\pi) .
$$

Ferner setzen wir voraus, daß durch die Störung die Entartung der angeregten Zustände aufgehoben wird. Lediglich für die angeregten B-Ringe in Benzol ist anzunehmen, daß die Aufspaltung der Energieniveaus gering ist.

Mit (V1) bis (V3) erhalten wir z. B. für die $\pi$-Elektronen des Anthracens das in Abb. 1 angegebene Energieniveau-Schema.

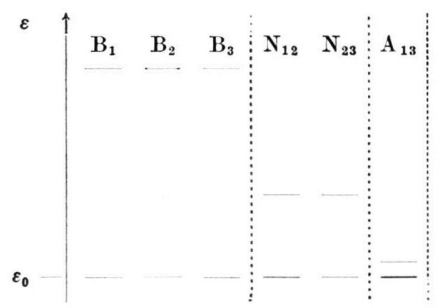

Abb. 1. Energieniveau-Schema für die $\pi$-Elektronen in Anthracen.

Die Funktionen $(\pi)$ und $\left(\pi^{\prime}\right)$ müssen wir, da die Beschränkung der Ausbreitungsmöglichkeit der $\pi$-Elektronen auf einzelne Ringe nur im effektiven Molekülfeld zustande kommen kann, als Eigenlösungen des Hamilton-Operators

$$
H_{i}=K_{i}+\sum_{j=1}^{M} V_{i j}+\sum_{\substack{k=1 \\ k \neq i}}^{N} W_{i k}
$$

( $K_{i}=$ Operator der kinetischen Energie eines $\pi$-Elektrons, $V_{i j}=$ Operator bzw. Potential der Wechselwirkung zwischen dem $i$-ten $\pi$-Elektron und dem $j$-ten $\mathrm{C}^{+}$Atomrumpf, $W_{i k}=$ Operator bzw. Potential der Wechselwirkung zwischen dem $i$-ten und dem $k$-ten $\pi$-Elektron, $M=$ Anzahl der C-Atome, $N=$ Anzahl der $\pi$-Elektronen) ansehen. $\varepsilon(\pi), \varepsilon\left(\pi^{\prime}\right)$ sind die zugehörigen Eigenwerte.

Von der Aufenthaltswahrscheinlichkeit eines $\pi$ Elektrons längs eines $\pi$-Ringes nehmen wir an, daß diese annähernd überall gleich sei, so daß, wenn $C_{v}, C_{v+1}$ zwei benachbarte Eckpunkte eines $\pi$-Ringes darstellen,

$$
\int_{C_{v}}^{C_{v+1}}|(\pi)|^{2} \mathrm{~d} x_{\pi}=\int_{C_{v}}^{C_{\nu+1}}\left|\left(\pi^{\prime}\right)\right|^{2} \mathrm{~d} x_{\pi}=\frac{1}{L(\pi)}
$$

wird. Die mittlere Dichte eines $\pi$-Elektrons soll also auf jeder Teilstrecke $C_{v} C_{v+1}$ eines $\pi$-Ringes

$$
\varrho=1 / L(\pi)
$$

betragen.

\section{Das System der $\pi$-Elektronen}

Der Hamilton-Operator $H$ des Systems der $\pi$ Elektronen ist mit (3) gegeben durch

$$
H=\sum_{i=1}^{N} H_{i}-\frac{1}{2} \sum_{\substack{i=1 \\(i \neq k)}}^{N} \sum_{\substack{k=1 \\ N}}^{N} W_{i k} .
$$

Die Eigenfunktionen $\Phi$ dieses Operators setzen wir als Produkt aus den Einelektronen-Eigenfunktionen des Abschnittes II/1 an. Vom Austausch sehen wir ab.

Von der Eigenfunktion $\Phi_{0}$ des Grundzustandes des Systems fordern wir:

(V5) $\Phi_{0}$ besitze die Symmetrie des Moleküls,

(V6) jede Einelektronen-Eigenfunktion $\left(\pi_{i}\right)$ sei in $\Phi_{0}$ mit "positivem“ und „negativem" Spin vertreten.

Zur Abkürzung schreiben wir

$$
\begin{aligned}
& \Phi_{0}=\left(\pi_{1}+\right)\left(\pi_{2}+\right)\left(\pi_{3}+\right) \ldots\left(\pi_{N / 2}+\right) \\
& \cdot\left(\pi_{1}-\right)\left(\pi_{2}-\right)\left(\pi_{3}-\right) \ldots\left(\pi_{N / 2}-\right) \\
& \equiv\left\{\left(\pi_{1}\right)\left(\pi_{2}\right)\left(\pi_{3}\right) \ldots\left(\pi_{N / 2}\right)\right\} .
\end{aligned}
$$

Die Energie $E$ des Systems ist durch den Hartreeschen Ausdruck ${ }^{7}$ gegeben:

$$
\left.\begin{array}{c}
E=\sum_{l=1}^{N} \varepsilon\left(\pi_{l}\right)-C ; \quad C \equiv \frac{1}{2} \sum_{\substack{i=1 \\
(i \neq k)}}^{N} \sum_{\substack{k=1 \\
(i \neq k)}}^{N} C_{i k} ; \\
C_{i k} \equiv \int_{0}^{L\left(\pi_{i}\right)} \int_{0}^{L\left(\pi_{k}\right)} W\left(x_{\pi i} ; x_{\pi_{k}}\right)\left|\left(\pi_{i}\right)\right|^{2}\left|\left(\pi_{k}\right)\right|^{2} \mathrm{~d} x_{\pi_{k}} \mathrm{~d} x_{\pi_{i}} .
\end{array}\right\}
$$

7 Vgl. etwa P. GoмвÁs, Theorie und Lösungsmethoden des Mehrteilchenproblems der Wellenmechanik, Verlag Birkhäuser, Basel 1950. 
Spalten wir die Integrale $C_{i k}$ auf in eine Doppelsumme über die einzelnen Teilstrecken $C_{\nu} C_{v+1}$ der Ringe $\pi_{i}$ und $\pi_{k}$ :

$$
C_{i k}=\sum_{v} \int_{C_{v}}^{C_{\nu+1}} \sum_{\mu}^{C_{\mu+1}} \int_{C_{\mu}}^{C_{\mu}} W\left(x_{\pi_{i}} ; x_{\pi_{k}}\right)\left|\left(\pi_{i}\right)^{2}\right|\left(\pi_{k}\right)^{2} \mathrm{~d} x_{\pi_{k}} \mathrm{~d} x_{\pi_{i}}
$$

und nehmen an, daß hierin alle Glieder mit $\nu \neq \mu$ zu vernachlässigen sind, setzen wir ferner in die dann noch verbleibenden Teilintegrale für $\left|\left(\pi_{j}\right)\right|^{2}$ den durch (V4) gegebenen mittleren Wert ein, so erhalten wir in erster Näherung

$$
C_{i k}=\frac{M\left(\pi_{i} ; \pi_{k}\right)}{L\left(\pi_{i}\right) L\left(\pi_{k}\right)} c \equiv\left[\left(\pi_{i}\right) ;\left(\pi_{k}\right)\right] .
$$

$M\left(\pi_{i} ; \pi_{k}\right)$ ist die Anzahl derjenigen Teilstrecken $\overline{C_{\nu} C_{v+1}}$ der Ringe $\pi_{i}$ und $\pi_{k}$, die sich überdecken,

$$
c \equiv \int_{C_{v}}^{C_{v+1}} \int_{C_{v}}^{C_{v+1}} W\left(x_{\tau_{i}} ; x_{n_{k}}\right) \mathrm{d} x_{\pi_{k}} \mathrm{~d} x_{\pi_{i}}
$$

hat auf allen Teilstrecken $C_{\nu} C_{\nu+1}$ den gleichen Wert und ist von den Indizes $i, k$ unabhängig.

Für Anthracen sind die Werte der Klammerausdrücke $\left[\left(\pi_{i}\right) ;\left(\pi_{k}\right)\right]$ in Tab. 1 angegeben.

\section{Der Grundzustand des Systems der శ-Elektronen in Acenen und Phenen}

Wie in Abschnitt I dargelegt wurde, beschränken wir die Ausbreitungsmöglichkeiten der $\pi$-Elektronen in Acenen und Phenen auf B-, N-... H-Ringe. Die Länge $L_{\max }$ des größten, in einem Molekül möglichen $\pi$-Ringes ist durch die maximale Anzahl $k$ der in diesem Molekül linear annellierten Benzolringe vorgeschrieben: $L_{\max }=4 k+2$.

Zur Bestimmung des Grundzustandes des Systems $\operatorname{der} \pi$-Elektronen stellen wir für jedes Molekül nach (V1) bis (V3) ein Energieniveau-Schema auf (vgl. Abb. 1) und besetzen dieses unter Berücksichtigung von (V5) und (V6) von unten auf.

Für Anthracen z. B. erhalten wir auf diese Weise als Grundzustand

$$
\Phi_{0}=\left\{\left(\mathrm{B}_{1}\right)\left(\mathrm{B}_{2}\right)\left(\mathrm{B}_{3}\right)\left(\mathrm{N}_{12}\right)\left(\mathrm{N}_{23}\right)\left(\mathrm{A}_{13}\right)\left(\mathrm{A}_{13}^{\prime}\right)\right\} .
$$

Die Energie $E_{0}$ dieses Grundzustandes errechnet man aus (4) zu

$$
E_{0}=14 \varepsilon_{0}+2 \Delta \varepsilon\left(\mathrm{A}_{13}^{\prime}\right)-C,
$$

\begin{tabular}{|c|c|c|c|c|c|c|}
\hline$\left(\pi_{i}\right)$ & $\left(\pi_{k}\right)$ & $L\left(\pi_{i}\right)$ & $L\left(\pi_{k}\right)$ & $\begin{array}{l}\overline{\vec{k}} \\
\ddot{\tilde{E}}\end{array}$ & \multicolumn{2}{|c|}{$\left[\left(\pi_{i}\right) ;\left(\pi_{k}\right)\right] / c$} \\
\hline $\begin{array}{l}\left(\mathrm{B}_{1}\right) \\
\left(\mathrm{B}_{2}\right) \\
\left(\mathrm{B}_{3}\right)\end{array}$ & $\begin{array}{l}\left(\mathrm{B}_{1}\right) \\
\left(\mathrm{B}_{2}\right) \\
\left(\mathrm{B}_{3}\right)\end{array}$ & 6 & 6 & 6 & $\begin{array}{c}6 \\
6 \cdot 6\end{array}$ & $=0,16667$ \\
\hline $\begin{array}{l}\left(\mathbf{N}_{12}\right) \\
\left(\mathbf{N}_{23}\right)\end{array}$ & $\begin{array}{l}\left(\mathbf{N}_{12}\right) \\
\left(\mathbf{N}_{23}\right)\end{array}$ & 10 & 10 & 10 & $\frac{10}{10 \cdot 10}$ & $=0,10000$ \\
\hline $\begin{array}{l}\left(\mathbf{B}_{1}\right) \\
\left(\mathbf{B}_{2}\right) \\
\left(\mathbf{B}_{2}\right) \\
\left(\mathbf{B}_{3}\right)\end{array}$ & $\begin{array}{l}\left(\mathbf{N}_{12}\right) \\
\left(\mathbf{N}_{12}\right) \\
\left(\mathbf{N}_{23}\right) \\
\left(\mathbf{N}_{23}\right)\end{array}$ & 6 & 10 & 5 & $\frac{5}{6 \cdot 10}$ & $=0,08333$ \\
\hline $\begin{array}{l}\left(A_{13}\right) \\
\left(A_{13}^{3}\right) \\
\left(A_{13}\right)\end{array}$ & $\begin{array}{l}\left(\mathrm{A}_{13}\right) \\
\left(\mathrm{A}_{13}^{\prime}\right) \\
\left(\mathrm{A}_{13}^{3}\right)\end{array}$ & 14 & 14 & 14 & $\begin{array}{c}14 \\
14 \cdot 14\end{array}$ & $=0,07143$ \\
\hline $\begin{array}{l}\left(\mathbf{N}_{12}\right) \\
\left(\mathbf{N}_{12}\right) \\
\left(\mathbf{N}_{23}\right) \\
\left(\mathbf{N}_{23}\right)\end{array}$ & $\begin{array}{l}\left(\mathrm{A}_{13}\right) \\
\left(\mathrm{A}_{13}^{\prime}\right) \\
\left(\mathrm{A}_{13}\right) \\
\left(\mathrm{A}_{13}^{3}\right)\end{array}$ & 10 & 14 & 9 & $\begin{array}{c}9 \\
10 \cdot 14\end{array}$ & $=0,06428$ \\
\hline $\begin{array}{l}\left(\mathrm{B}_{1}\right) \\
\left(\mathrm{B}_{1}\right) \\
\left(\mathrm{B}_{3}\right) \\
\left(\mathrm{B}_{3}\right)\end{array}$ & $\begin{array}{l}\left(\mathrm{A}_{13}\right) \\
\left(\mathrm{A}_{13}^{\prime}\right) \\
\left(\mathrm{A}_{13}\right) \\
\left(\mathrm{A}_{13}^{\prime}\right)\end{array}$ & 6 & 14 & 5 & $\begin{array}{c}5 \\
6 \cdot 14\end{array}$ & $=0,05952$ \\
\hline $\begin{array}{l}\left(\mathrm{B}_{2}\right) \\
\left(\mathrm{B}_{2}\right)\end{array}$ & $\begin{array}{l}\left(\mathrm{A}_{13}\right) \\
\left(\mathrm{A}_{13}^{\prime}\right)\end{array}$ & 6 & 14 & 4 & $\frac{4}{6 \cdot 14}$ & $=0,04762$ \\
\hline$\left(\mathrm{N}_{12}\right)$ & $\left(\mathrm{N}_{23}\right)$ & 10 & 10 & 4 & $\frac{4}{10 \cdot 10}$ & $=0,04000$ \\
\hline $\begin{array}{l}\left(\mathrm{B}_{1}\right) \\
\left(\mathrm{B}_{2}\right)\end{array}$ & $\begin{array}{l}\left(\mathrm{B}_{2}\right) \\
\left(\mathrm{B}_{3}\right)\end{array}$ & 6 & 6 & 1 & $\frac{1}{6 \cdot 6}$ & $=0,02778$ \\
\hline $\begin{array}{l}\left(\mathrm{B}_{1}\right) \\
\left(\mathrm{B}_{3}\right)\end{array}$ & $\begin{array}{l}\left(\mathrm{N}_{23}\right) \\
\left(\mathbf{N}_{12}\right)\end{array}$ & 6 & 10 & 1 & $\frac{1}{6 \cdot 10}$ & $=0,01667$ \\
\hline$\left(B_{1}\right)$ & $\left(\mathrm{B}_{3}\right)$ & 6 & 6 & 0 & & 0,00000 \\
\hline
\end{tabular}

wobei nach (4) und (5) $C=5,339 c$ wird.

Die Grundzustände und $C$-Werte für einige andere Moleküle sind in Tab. 2 angegeben.
Tab. 1 .

Allgemein ergibt sich, daß die Energien $E_{0}$ der Grundzustände aus drei Einzelbeträgen zusammengesetzt sind:

1. Einem Betrag, der sich additiv aus konstanten Beiträgen der einzelnen $\pi$-Elektronen zusammensetzt (bei Anthracen zusammen $14 \varepsilon_{0}$ ),

2. den Anregungsenergien etwa vorhandener angeregter $\pi$-Elektronen [bei Anthracen $\left.2 \Delta \varepsilon\left(\mathrm{A}_{13}^{\prime}\right)\right]$,

3. einem Betrag $C$, hervorgerufen durch die Wechselwirkung der $\pi$-Elektronen untereinander.

Da die Energien $E_{0}$, bis auf eine additive Konstante, mit dem Energiebetrag übereinstimmen müssen, den das System der $\pi$-Elektronen zur Bindungsenergie der Moleküle beisteuert, erhalten wir auch für den Beitrag $E_{\mathrm{B}}$ der $\pi$-Elektronen zur Bindungsenergie diese Aufspaltung. Dies steht im Einklang mit der Erfahrung, daß $E_{\mathrm{B}}$ darstellbar ist durch

$$
E_{\mathrm{B}}=-E_{\mathrm{K}}-E_{\mathrm{s}} \text {, }
$$


wobei $E_{\mathrm{K}}$ additiv aus konstanten Beiträgen der einzelnen $\pi$-Elektronen zusammengesetzt ist und $E_{\mathrm{s}}$ die sog. Sonderenergie bedeutet ${ }^{8}$. $E_{\mathrm{s}}$ ist also nach unserem Verfahren durch die Coulombsche Wechselwirkung der $\pi$-Elektronen untereinander und durch die Anregungsenergien der $\pi$-Elektronen gegeben. Für Anthracen erhalten wir z. B.

$$
E_{\mathrm{s}}=C-2 \Delta \varepsilon\left(\mathrm{A}_{13}^{\prime}\right) \text {. }
$$

Nehmen wir für $\gamma$ in (V3) einen Wert in der Größenordnung von 1 bis $15 \mathrm{eV}$ oder $20 \mathrm{bis} 300 \mathrm{kcal} / \mathrm{Mol}$ an (weiter unten wird sich diese Annahme als notwendig erweisen, um mit der Erfahrung in Übereinstimmung zu bleiben), so können wir die Anregungsenergien bei den meisten Molekülen gegenüber $C$ vernachlässigen. In erster Näherung ist dann die Sonderenergie durch $C$ gegeben.

Ein Vergleich mit den experimentell bekannten Sonderenergien (für Anthracen z. B. ist $E_{\mathrm{s}}=116$ $\mathrm{kcal} / \mathrm{Mol}, \mathrm{s.}$ Anm. ${ }^{8}$ ) zeigt dann, daß $c$ etwa gleich $22 \mathrm{kcal} / \mathrm{Mol} \approx 1 \mathrm{eV}$ sein $\mathrm{mu}$.

\section{Vergleich der C-Werte mit den HückeLschen Sonderenergien}

Die Bindungsenergien bzw. Sonderenergien der meisten Aromaten sind experimentell nicht bestimmt. Wir sind daher, um unser Modell zu prüfen, in der Mehrzahl der Fälle darauf angewiesen, andere Daten zum Vergleich heranzuziehen. Hier bieten sich vor allem die mit der Hückelschen Methode ${ }^{9}$ berechneten Werte der Sonderenergien an, die, soweit ein Vergleich möglich ist, gut mit der Erfahrung übereinstimmen.

Bei Hückel werden nun die Sonderenergien als Vielfache eines Resonanzintegrals $\beta$ angegeben und bemerkenswert ist zunächst, daß der Wert unseres Wechselwirkungsintegrals $c \approx 20 \mathrm{kcal} / \mathrm{Mol}$ mit dem Betrag von $\beta$ übereinstimmt. Man kann zeigen, da $\beta$ diese Übereinstimmung plausibel wird, wenn die bei der HücKelschen Methode verwendeten $(2 \mathrm{p} \pi)$. Atomeigenfunktionen sich weitgehend überlappen. Eine derartige Überlappung entspricht aber gerade der Grundvoraussetzung unseres Modells. Zu beachten ist bei dem Vergleich von $c$ und $|\beta|$, da $\beta c$, so wie es in Abschnitt II/2 angegeben wurde, nur einen formellen Sinn hat. Mit

$$
W\left(x_{\pi_{i}} ; x_{\pi_{k}}\right)=e_{0}{ }^{2} / l_{0} \cdot r\left(x_{\pi_{i}} ; x_{\pi_{k}}\right)
$$

$\left(e_{0}=\right.$ Elementarladung; $r\left(x_{\pi_{i}} ; x_{\pi_{k}}\right)=$ Abstand des $i$-ten vom $k$-ten $\pi$-Elektron) wird nämlich $c$ divergent. Dies liegt jedoch lediglich daran, daß wir dort für das dreidimensionale Problem eine eindimensionale Symbolik verwenden. Bei der Durchrechnung des Problems mit dreidimensionalen Dichteverteilungen wird $c$ die Coulombsche Wechselwirkung zweier Elektronen, die in einem quaderförmigen Kasten der Länge $L=1$, der Breite $B<1$ und der Höhe $H<1$,eingesperrt" sind. Die eindimensionalen Dichtefunktionen $\psi\left(x_{\pi}\right)$ sind dabei zu einer dreidimensionalen Dichtefunktion $\psi\left(x_{\pi} ; y ; z\right)=\psi\left(x_{\pi}\right) \cdot \varphi(y ; z) \quad(y ; z=$ Koordinaten in Richtung der Quaderkanten B und H) zu ergänzen, wobei nach KuHN ${ }^{10}$

$$
\varphi(y ; z)=\left[\frac{4}{B H}\right]^{1 / 2} \sin \left(\varkappa \frac{y}{B}\right) \sin \left(2 \varkappa \frac{z}{H}\right)
$$

gesetzt werden kann.

In Tab. 2, Spalte 5, sind die mit der Hückelschen Methode errechneten Sonderenergien in Einheiten von $|\beta|$ angegeben $^{8}$. Ein Vergleich unserer $C$-Werte mit den Hückelschen Sonderenergien zeigt bei den meisten Molekülen eine Übereinstimmung bis auf $2 \%$.

Die Abweichungen unserer $C$-Werte von den Hückelschen Sonderenergien bei Benzol und Naphthalin sind damit zu erklären, daß in diesen Molekülen angeregte B-Elektronen vertreten sind, deren Anregungsenergie nach (V3) relativ hoch über der Energie der nicht angeregten Elektronen liegt, so daß hier $E_{\mathrm{s}}<C$ wird. Bei Benzol müssen wir übrigens, um alle $\pi$-Elektronen unterbringen zu können, noch die Existenz eines zweiten angeregten Zustandes $\left(B^{\prime \prime}\right)$ voraussetzen. Für die Energie dieses $\mathrm{Zu}$ standes gilt $\varepsilon\left(\mathrm{B}^{\prime \prime}\right)>\varepsilon\left(\mathrm{B}^{\prime}\right)$. Da jedoch die $\pi$-Elektronen in Benzol keiner asymmetrischen Störung durch Nachbar-Ringe ausgesetzt sind, können wir annehmen, $\mathrm{da} \beta \varepsilon\left(\mathrm{B}^{\prime \prime}\right)$ sich nicht wesentlich von $\varepsilon\left(\mathrm{B}^{\prime}\right)$ unterscheidet (vgl. auch Tab. 3).

Ein großer Unterschied zwischen der Hückelschen Sonderenergie und unserem $C$-Wert tritt bei Pentacen auf. Hier, wie bei Hexacen, erweist sich unser Verfahren mit den einfachen Annahmen (V1) - (V3) bei der Bestimmung der Energie des Grundzustandes als nicht ausreichend: Bei gleicher (minimaler) Summe $\Sigma \varepsilon\left(\pi_{l}\right)=N \varepsilon_{0}$ aus (4) ergeben sich mehrere

\footnotetext{
8 Vgl. etwa H. Hartmans, Theorie der chemischen Bindung auf quantentheoretischer Grundlage, Springer-Verlag, Berlin 1954, Abschn. 1511.

9 Eine Übersicht über diese Methode und Literaturangaben finden sich in H. Hartmann, 1. c. ${ }^{8}$, Abschn. 155.

10 H. Kuns, Experientia 9, 2 [1953].
} 


\begin{tabular}{|c|c|c|c|c|}
\hline Molekül & Struktur & Besetzung $\Phi_{0}$ & $\begin{array}{c}C \\
{[\text { Einheit }} \\
c]\end{array}$ & $\begin{array}{l}\text { HÜCKELsche } \\
\text { Sonderenergif } \\
\text { [Einheit }|\boldsymbol{\beta}|]\end{array}$ \\
\hline Benzol & & $\left\{(\mathrm{B})\left(\mathrm{B}^{\prime}\right)\left(\mathrm{B}^{\prime \prime}\right)\right\}$ & 2,500 & 2,000 \\
\hline Naphthalin & 2 & $\left\{\left(\mathrm{~B}_{1}\right)\left(\mathrm{B}_{1}^{\prime}\right)\left(\mathrm{B}_{2}\right)\left(\mathrm{B}_{2}^{\prime}\right)\left(\mathrm{N}_{12}\right)\right\}$ & 3,878 & 3,683 \\
\hline Anthracen & & $\left\{\left(\mathrm{B}_{1}\right)\left(\mathrm{B}_{2}\right)\left(\mathrm{B}_{3}\right)\left(\mathrm{N}_{12}\right)\left(\mathrm{N}_{23}\right)\left(\mathrm{A}_{13}\right)\left(\mathrm{A}_{13}^{\prime}\right)\right\}$ & 5,339 & 5,319 \\
\hline Tetracen & & $\left\{\left(\mathrm{B}_{1}\right)\left(\mathrm{B}_{2}\right)\left(\mathrm{B}_{3}\right)\left(\mathrm{B}_{4}\right)\left(\mathrm{N}_{12}\right)\left(\mathrm{N}_{23}\right)\left(\mathrm{N}_{34}\right)\left(\mathrm{A}_{13}\right)\left(\mathrm{A}_{24}\right)\right\}$ & 6,918 & 6,932 \\
\hline Pentacen & & $\begin{array}{l}\left\{\left(\mathrm{B}_{2}\right)\left(\mathrm{B}_{3}\right)\left(\mathrm{B}_{4}\right)\left(\mathrm{N}_{23}\right)\left(\mathrm{N}_{34}\right)\left(\mathrm{A}_{13}\right)\left(\mathrm{A}_{24}\right)\left(\mathrm{A}_{35}\right)\right. \\
\left.\quad\left(\mathrm{R}_{14}\right)\left(\mathrm{R}_{25}\right)\left(\mathrm{P}_{15}\right)\right\}\end{array}$ & 10,642 & 8,544 \\
\hline Hexacen & & $\begin{array}{l}\left\{\left(\mathrm{B}_{3}\right)\left(\mathrm{B}_{4}\right)\left(\mathrm{N}_{23}\right)\left(\mathrm{N}_{34}\right)\left(\mathrm{N}_{45}\right)\left(\mathrm{A}_{24}\right)\left(\mathrm{A}_{35}\right)\left(\mathrm{R}_{14}\right)\right. \\
\left.\quad\left(\mathrm{R}_{25}\right)\left(\mathrm{R}_{36}\right)\left(\mathrm{P}_{15}\right)\left(\mathrm{P}_{26}\right)\left(\mathrm{H}_{16}\right)\right\}\end{array}$ & 13,899 & - \\
\hline Phenanthren & & $\left\{\left(\mathrm{B}_{1}\right)\left(\mathrm{B}_{2}\right)\left(\mathrm{B}_{3}\right)\left(\mathrm{N}_{12}\right)\left(\mathrm{N}_{12}^{\prime}\right)\left(\mathrm{N}_{23}\right)\left(\mathrm{N}_{23}^{\prime}\right)\right\}$ & 5,496 & 5,448 \\
\hline Tetraphen & & $\left\{\left(\mathrm{B}_{1}\right)\left(\mathrm{B}_{2}\right)\left(\mathrm{B}_{3}\right)\left(\mathrm{B}_{4}\right)\left(\mathrm{N}_{12}\right)\left(\mathrm{N}_{23}\right)\left(\mathrm{N}_{34}\right)\left(\mathrm{A}_{13}\right)\left(\mathrm{A}_{13}^{\prime}\right)\right\}$ & 7,041 & 7,101 \\
\hline Pentaphen & & $\begin{array}{l}\left\{\left(\mathrm{B}_{1}\right)\left(\mathrm{B}_{2}\right)\left(\mathrm{B}_{3}\right)\left(\mathrm{B}_{4}\right)\left(\mathrm{B}_{5}\right)\left(\mathrm{N}_{12}\right)\left(\mathrm{N}_{23}\right)\left(\mathrm{N}_{34}\right)\right. \\
\left.\quad\left(\mathrm{N}_{45}\right)\left(\mathrm{A}_{13}\right)\left(\mathrm{A}_{35}\right)\right\}\end{array}$ & 8,272 & 8,763 \\
\hline $\begin{array}{l}\text { 1.2,5.6- } \\
\text { Dibenz- } \\
\text { anthracen }\end{array}$ & & $\begin{array}{l}\left\{\left(\mathrm{B}_{1}\right)\left(\mathrm{B}_{2}\right)\left(\mathrm{B}_{3}\right)\left(\mathrm{B}_{4}\right)\left(\mathrm{B}_{5}\right)\left(\mathrm{N}_{12}\right)\left(\mathrm{N}_{23}\right)\left(\mathrm{N}_{34}\right)\left(\mathrm{N}_{45}\right)\right. \\
\left.\quad\left(\mathrm{A}_{24}\right)\left(\mathrm{A}_{24}^{\prime}\right)\right\}\end{array}$ & 8,742 & 8,880 \\
\hline Picen & & $\begin{array}{l}\left\{\left(\mathrm{B}_{1}\right)\left(\mathrm{B}_{2}\right)\left(\mathrm{B}_{3}\right)\left(\mathrm{B}_{4}\right)\left(\mathrm{B}_{5}\right)\left(\mathrm{N}_{12}\right)\left(\mathrm{N}_{23}\right)\left(\mathrm{N}_{23}^{\prime}\right)\left(\mathrm{N}_{34}\right)\right. \\
\left.\quad\left(\mathrm{N}_{34}^{\prime}\right)\left(\mathrm{N}_{45}\right)\right\}\end{array}$ & 8,784 & 8,943 \\
\hline $\begin{array}{l}\text { 1.2, 3.4- } \\
\text { Dibenz- } \\
\text { anthracen }\end{array}$ & & $\begin{array}{l}\left\{\left(\mathrm{B}_{1}\right)\left(\mathrm{B}_{2}\right)\left(\mathrm{B}_{3}\right)\left(\mathrm{B}_{4}\right)\left(\mathrm{B}_{5}\right)\left(\mathrm{N}_{12}\right)\left(\mathrm{N}_{23}\right)\left(\mathrm{N}_{34}\right)\left(\mathrm{N}_{35}\right)\right. \\
\left.\quad\left(\mathrm{A}_{13}\right)\left(\mathrm{A}_{13}^{\prime}\right)\right\}\end{array}$ & 9,036 & 8,942 \\
\hline
\end{tabular}

Tab. 2 . 


\begin{tabular}{|c|c|c|c|c|c|c|c|}
\hline \multirow{2}{*}{$z$} & \multirow{2}{*}{ Struktur } & \multicolumn{2}{|c|}{ Anz. d. angeregten } & \multirow{2}{*}{$\begin{array}{c}C \\
{\left[\begin{array}{c}\text { Einheit } \\
c]\end{array}\right.}\end{array}$} & \multirow{2}{*}{$\begin{array}{l}\sum_{l} \Delta \varepsilon\left(\pi_{l}^{\prime}\right) \\
{[\text { Einheit } c]}\end{array}$} & \multirow{2}{*}{$\begin{array}{c}C-\sum_{l} \Delta \varepsilon\left(\pi_{l}^{\prime}\right) \\
{[\text { Einheit } c]}\end{array}$} & \multirow{2}{*}{$\begin{array}{l}\text { HUtckELsche } \\
\text { Sonderenergie } \\
{[\text { Einheit } \beta \mid}\end{array}$} \\
\hline & & B-Ringe & D-Ringe & & & & \\
\hline 1 & & 4 & 0 & 2,500 & 0,500 & 2,000 & 2,000 \\
\hline 2 & & 4 & 2 & 4,923 & 0,540 & 4,383 & 4,383 \\
\hline 3 & & 4 & 4 & 7,350 & 0,580 & 6,770 & 6,772 \\
\hline 4 & & 4 & 6 & 9,777 & 0,620 & 9,157 & 9,160 \\
\hline 5 & & 4 & 8 & 12,204 & 0,660 & 11,544 & - \\
\hline
\end{tabular}

Tab. 3 .

Möglichkeiten der Besetzung des EnergieniveauSchemas. Unter diesen müssen wir diejenige als Grundzustand ansehen, für die $E$ den kleinsten Wert annimmt. Wegen $\Sigma \varepsilon\left(\pi_{l}\right)=$ const ist das die Besetzung, für die $C$ den größten Wert hat. $\mathrm{Zu}$ beachten ist dann, daß $C$ um so größer wird, je mehr sich die einzelnen $\pi$-Ringe überlappen. Dadurch ergibt sich für die angegebenen Grundzustände von Pentacen und Hexacen eine derart ungleichmäßige Verteilung der $\pi$-Elektronen in diesen Molekülen, daß unsere Voraussetzung (V2), $\varepsilon\left(\pi_{l}\right)$ sei konstant $=\varepsilon_{0}$, sicher nicht mehr erfüllt ist. In diesen Fällen wäre es also notwendig, die durch die ungleichmäßige Verteilung der $\pi$-Elektronen hervorgerufene Änderung der Lage der Energieniveaus $\varepsilon\left(\pi_{l}\right)$ für jede zu $\Sigma \varepsilon\left(\pi_{l}\right)=N \varepsilon_{0}$ mögliche Besetzung durch einen zweiten Näherungsschritt (etwa durch den ersten Schritt der Methode des self consistent field) zu bestimmen.

Weiterhin erhalten wir für Pentaphen einen etwas zu kleinen $C$-Wert ${ }^{11}$. Ebenso bei Triphenylen $\left[C=7,020 c ; E_{\mathrm{s}}\right.$ (HüскеL) $\left.=7,275|\beta|\right]$ und Chrysen $\left[C=6,833 c ; E_{\mathrm{s}} \quad\right.$ (Hückel $\left.)=7,190|\beta|\right]$, die beide, wie experimentell erwiesen ${ }^{8}$, energetisch günstiger liegen als Tetracen und Tetraphen.

Ein Vergleich der Verbrennungswärmen von Tetracen und Tetraphen zeigt übrigens, daß die Anregungsenergien $\Delta \varepsilon\left(\pi^{\prime}\right)$ von Bedeutung sein können, auch wenn sie gegenüber $C$ klein sind. Für die Energie des Systems der $\pi$-Elektronen dieser Moleküle erhalten wir nach unserer Methode

für Tetracen $E_{0}=18 \varepsilon_{0}-6,918 c$,

für Tetraphen $E_{0}=18 \varepsilon_{0}+2 \Delta \varepsilon\left(\mathrm{A}_{13}^{\prime}\right)-7,041 c$.

Der Vergleich der gemessenen Verbrennungswärmen zeigt nun, daß Tetraphen energetisch um $0,8 \mathrm{kcal}$ pro Mol günstiger liegt als Tetracen ${ }^{8}$. Da die additiven Beiträge zur Bindungsenergie bei beiden Molekülen die gleichen sind, kann der Energieunterschied nur in den Sonderenergien auftreten. Die Differenz der Sonderenergien beträgt jedoch nach HückeL $3,4 \mathrm{kcal} / \mathrm{Mol}$, fällt also zu groß aus. Nach unserer Methode erhält man als Differenz der Sonderenergien

$$
2,5 \mathrm{kcal} / \mathrm{Mol}-2 \Delta \varepsilon\left(\mathrm{A}_{13}^{\prime}\right) .
$$

Bei einer Vernachlässigung der Anregungsenergie $2 \Delta \varepsilon\left(\mathrm{A}_{13}^{\prime}\right)$ gegenüber $C \approx 140 \mathrm{kcal} / \mathrm{Mol}$ würde auch unser Wert zu groß ausfallen. Nehmen wir jedoch $\Delta \varepsilon\left(\mathrm{A}_{13}^{\prime}\right)=0,85 \mathrm{kcal} / \mathrm{Mol}$ [bzw. in (V3) $\gamma=166$ $\mathrm{kcal} / \mathrm{Mol} \approx 7,5 \mathrm{eV}]$ an, so erhalten wir volle Übereinstimmung mit der Erfahrung.

Bemerkenswert ist schließlich, daß auch mit unserem Modell der große Unterschied der Verbrennungswärmen von Anthracen und Phenanthren, etwa $14 \mathrm{kcal} / \mathrm{Mol}^{8}$, nicht erklärt werden kann. Wir erhalten vielmehr, wie mit der HückELschen und Paulingschen Methode ${ }^{12}$, nur einen Unterschied von etwa $3 \mathrm{kcal} / \mathrm{Mol}$.

\section{Die Verteilung der a-Elektronen in Acenen und Phenen}

Nach unserem Modell ist die Dichte des Systems der $\pi$-Elektronen auf den einzelnen Teilstrecken $C_{v} C_{v+1}$ zwischen zwei benachbarten $C$-Atomen $C_{\nu}$ und $C_{v+1}$ durch zwei Komponenten bestimmt:

11 Wenigstens gegenüber der Hückelschen Sonderenergie. Experimentelle Werte liegen hier nicht vor.

12 Vgl. auch W. HückeL, Theoretische Grundlagen der organischen Chemie, II, 7. Aufl., Akademische Verlagsgesellschaft Geest und Portig K.-G.. Leipzig 1954. 
1. Durch die „Verteilung“ des einzelnen $\pi$-Elektrons auf dem ihm zugeordneten $\pi$-Ring,

2. durch die Verteilung der mit Elektronen besetzten $\pi$-Ringe über das Molekül.

Wegen (V4) können wir mit unserem einfachen Verfahren nur die durch die zweite Komponente hervorgerufenen Dichteschwankungen längs des Molekülgerüsts erfassen.

Nach (V4) beträgt die mittlere Dichte $P$ des Systems der $\pi$-Elektronen auf einer Teilstrecke $C_{\nu} C_{v+1}$ :

$$
P=\sum_{l} 1 / L\left(\pi_{l}\right)
$$

(zu summieren ist über die Zahl der $\pi$-Elektronen, deren Dichte auf $C_{v} C_{v+1}$ von Null verschieden ist).

$P=0$ entspricht der einfachen $\mathrm{C}-\mathrm{C}$-Bindung, $P=1$ der aromatischen C-C-Bindung mit $50 \%$ Doppelbindungscharakter und $P=2$ der ungesättigten Doppelbindung.

Für die Acene errechnet man eine mit wachsender Annellierung steigende $\pi$-Elektronendichte in den mittleren Ringen (von Benzol $P=1$ bis Heptacen $P=1,85$ ), entsprechend der mit der Annellierung steigenden Reaktivität dieser Moleküle an den Mittelringen und entsprechend der wachsenden Beständigkeit z. B. der Dihydroacene ${ }^{13}$. Bei den Phenen zeigt sich in den Ringen, an denen die angulare Annellierung erfolgte, eine erhöhte $\pi$-Elektronendichte. Diese steigt relativ zur Dichte in den übrigen Ringen und im Vergleich zu den Acenen nur schwach mit wachsender Ringzahl an. Dem entspricht die erhöhte Reaktivität der Phene an diesen Ringen und die gegenüber den Acenen weit geringere Zunahme der Beständigkeit der Dihydrophene mit wachsender Annellierung ${ }^{13}$.

\section{Der Grundzustand des Systems der л-Elektronen in Polyphenylketten}

Um dem aromatischen Charakter der mittleren C-C-Bindung in Diphenyl Rechnung zu tragen, nehmen wir an, daß in Diphenyl die $\pi$-Elektronen sich, außer auf B-Ringen, auch längs eines Streckenzuges der Form

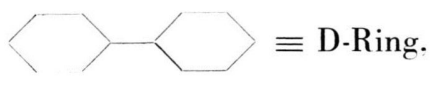

13 Vgl. etwa E. Clar, l. c. ${ }^{3}$, S. 10/11.

14 Bezeichnet $C_{a}$ den Wert von $C$ in (4), den man erhält, wenn man die restlichen vier $\pi$-Elektronen auf die äußeren B'$^{\prime}$-Ringe verteilt, $C_{i}$ den entsprechenden Wert bei Vertei- mit $L(D)=13$, ausbreiten können. Da die Wellenlängen im Absorptionsspektrum der Polyphenyle nur wenig mit wachsender Ringzahl zunehmen ${ }^{3}$, beschränken wir die Ausbreitungsmöglichkeiten der $\pi$-Elektronen auch in mehrgliedrigen Phenylketten auf B- und D-Ringe.

Für Diphenyl bzw. Terphenyl (vgl. Tab. 3, Spalte 2) erhalten wir so als Grundzustand

$$
\begin{aligned}
\Phi_{0}{ }^{(2)}=\{ & \left.\left(\mathrm{B}_{1}\right)\left(\mathrm{B}_{1}{ }^{\prime}\right)\left(\mathrm{B}_{2}\right)\left(\mathrm{B}_{2}{ }^{\prime}\right)\left(\mathrm{D}_{12}\right)\left(\mathrm{D}_{12}{ }^{\prime}\right)\right\} \quad \text { bzw. } \\
\Phi_{0}{ }^{(3)}=\left\{\left(\mathrm{B}_{1}\right)\left(\mathrm{B}_{1}{ }^{\prime}\right)\left(\mathrm{B}_{2}\right)\left(\mathrm{B}_{3}\right)\left(\mathrm{B}_{3}{ }^{\prime}\right)\left(\mathrm{D}_{12}\right)\left(\mathrm{D}_{12}{ }^{\prime}\right)\left(\mathrm{D}_{23}\right)\right. & \\
& \left.\left(\mathrm{D}_{23}{ }^{\prime}\right)\right\} .
\end{aligned}
$$

Für die höheren Polyphenyle ergeben sich bei gleicher (minimaler) Summe $\Sigma \varepsilon\left(\pi_{1}\right)$ aus (4) zwei Möglichkeiten der Besetzung: $\mathrm{Zu}$ den $\pi$-Ringen $\left(\mathrm{B}_{1}\right)\left(\mathrm{B}_{2}\right) \ldots\left(\mathrm{B}_{z}\right)$ und $\left(\mathrm{D}_{12}\right)\left(\mathrm{D}_{12}{ }^{\prime}\right)\left(\mathrm{D}_{23}\right)\left(\mathrm{D}_{23}{ }^{\prime}\right) \ldots$ $\left(\mathrm{D}_{z-1, z}\right)\left(D_{z-1, z^{\prime}}\right) \quad(z=$ Anzahl der Kettenglieder $)$ können entweder vier innere oder vier äußere angeregte B-Ringe $\left(\mathrm{B}_{i}{ }^{\prime}\right)$ bzw. $\left(\mathrm{B}_{a}{ }^{\prime}\right)$ besetzt werden. Die Besetzung $\left(\mathrm{B}_{1}\right)\left(\mathrm{B}_{2}\right) \ldots\left(\mathrm{B}_{z}\right)\left(\mathrm{D}_{12}\right)\left(\mathrm{D}_{12}^{\prime}\right) \ldots\left(\mathrm{D}_{z-1, z}\right)$ $\left(\mathrm{D}_{z-1, z}{ }^{\prime}\right)$ bewirkt nun in den inneren Gliedern eine gegenüber den äußeren Gliedern erhöhte $\pi$-Elektronendichte, so daß

$$
\varepsilon\left(\mathrm{B}_{a}{ }^{\prime}\right)<\varepsilon\left(\mathrm{B}_{i}{ }^{\prime}\right) \quad(a=1, z ; i=2,3, \ldots, z-1)
$$

ist. Besetzt man daher, um für den Grundzustand zu einem Energieminimum zu kommen, mit den restlichen vier $\pi$-Elektronen die äußeren $B^{\prime}$-Ringe ${ }^{14}$, so erhält man für alle Polyphenyle den Grundzustand

$$
\begin{aligned}
\Phi_{0}{ }^{(z)}= & \left\{\left(\mathrm{B}_{1}\right)\left(\mathrm{B}_{1}{ }^{\prime}\right)\left(\mathrm{B}_{2}\right)\left(\mathrm{B}_{3}\right)\right. \\
& \ldots\left(\mathrm{B}_{z-1}\right)\left(\mathrm{B}_{z}\right)\left(\mathrm{B}_{z}{ }^{\prime}\right)\left(\mathrm{D}_{12}\right)\left(\mathrm{D}_{12}{ }^{\prime}\right) \\
& \left.\ldots\left(\mathrm{D}_{z-1, z}\right)\left(\mathrm{D}_{z-1, z}^{\prime}\right)\right\} ;
\end{aligned}
$$

$(z>1)$. Jeweils die äußersten B-Ringe und alle D-Ringe sind darin mit angeregten Zuständen vertreten. Für den zu $\Phi_{0}{ }^{(z)}$ gehörigen $C$-Wert erhält man

$$
C=(2,427 z+0,069) c
$$

(vgl. Tab. 3, Spalte 4).

Eine praktisch vollkommene Übereinstimmung mit den nach der Hückelschen Methode errechneten Sonderenergien erhalten wir, wenn wir annehmen, daß

$$
\begin{aligned}
\Delta \varepsilon\left(\mathrm{B}_{1}{ }^{\prime}\right)=\Delta \varepsilon\left(\mathrm{B}_{z}{ }^{\prime}\right) & =0,125 c \quad \text { und } \\
\Delta \varepsilon\left(\mathrm{D}_{u,}{ }^{\prime}, \mu+1\right) & =0,020 c
\end{aligned}
$$

lung dieser Elektronen auf innere B'-Ringe, so wird, wegen der erhöhten Elektronendichte auf den inneren Gliedern, $C_{i}>C_{a}$. Wir nehmen also an, daß

$$
4\left[\varepsilon\left(\mathrm{B}_{i}{ }^{\prime}\right)-\varepsilon\left(\mathrm{B}_{a^{\prime}}\right)\right]>C_{i}-C_{a} \text { ist. }
$$


ist (Tab. 3, Spalte 6 und 7). Dies entspricht etwa dem Ansatz (V 3) mit $\gamma=4,5 c$.

Für die mittleren Dichten $P$ des Systems der $\pi$-Elektronen in Polyphenylen erhalten wir Werte um $P=1$. Lediglich für die zwischen den BenzolRingen gelegenen $\mathrm{C}-\mathrm{C}$-Bindungen wird $P=0,3$.

\section{Bemerkung über die Verteilung der r-Elektronen in Pyrenen}

Ein Vergleich der Absorptionsspektren der Pyrene untereinander und mit denen der Acene und Phene läßt zunächst in den Pyrenen eine Verteilung der $\pi$-Elektronen auf B-, N-, A-...-Ringe vermuten. Beschränken wir jedoch die Ausbreitungsmöglichkeiten der $\pi$-Elektronen z. B. in Pyren nur auf Bund N-Ringe, so erhalten wir einen Grundzustand, dessen $C$-Wert schlecht mit der von HücKel errechneten Sonderenergie ${ }^{8}$ übereinstimmt. Lassen wir dagegen mit CLAR ${ }^{15}$ in Pyren, neben den B- und $\mathrm{N}$-Ringen, auch einen D-Ring zu, so wird die Übereinstimmung mit HücKel gut.

Bei der Bestimmung des Grundzustandes der höher annellierten Pyrene ergeben sich, wie bei Pentacen und Hexacen, bei gleicher (minimaler) Summe $\Sigma \varepsilon\left(\pi_{l}\right)$ aus (4), mehrere Möglichkeiten der Besetzung des Energieniveau-Schemas. Bemerkenswert ist, daß wir für 3.4-Benzpyren, 1.2,3.4-Dibenzpyren und 3.4,8.9-Dibenzpyren, die stark cancerogen sind, für die als Grundzustand in Frage kommenden Besetzungen (großes $C$ ), eine besonders große $\pi$-Elektronendichte an denjenigen Stellen erhalten, die Druckrey ${ }^{16}$ für die cancerogene Aktivität dieser Moleküle verantwortlich macht.

\section{Schlußbetrachtung}

Das in Abschnitt II dargelegte einfache Ringmodell bedarf zweifellos an einigen Punkten noch einer Verbesserung. Dies war, nach der Annahme der recht groben Voraussetzungen $(\mathrm{V} 1)-(\mathrm{V} 6)$, nicht anders zu erwarten. Interessant ist, daß die Annahme der Verteilung der $\pi$-Elektronen auf einzelne Ringe sich zu bestätigen scheint und daß die sog. Sonderenergie der Aromaten, indem sie im wesentlichen sich aus der Coulombschen Wechselwirkung der $\pi$-Elektronen untereinander ergibt, auch „klassisch“ erklärbar wird.

Differenziertere Aussagen über die Verteilung der $\pi$-Elektronen und eine Erklärung der Absorptionsspektren der aromatischen Kohlenwasserstoffe kann man mit dem Ring-Modell nur erwarten, wenn die dem Hamilton-Operator (3) entsprechende Schrödinger-Gleichung explizit gelöst wird. Mit der Annahme einer freien Ausbreitung der $\pi$-Elektronen über die $\pi$-Ringe - das ist die einfachste Möglichkeit, zu einer expliziten Lösung der SchrödingerGleichung zu kommen - erhält man, wie zu erwarten, keinerlei Übereinstimmung mit der Erfahrung. Eine angenähert freie Ausbreitung der $\pi$-Elektronen kann man jedoch annehmen, wenn man die $\pi$-Elektronen allein im Feld der $\mathrm{C}^{+}$-Atomrümpfe betrachtet, die Lösungen (1) also als Eigenlösungen des Hamilton-Operators

$$
H_{i}=K_{i}+\sum_{j=1}^{M} V_{i j} \approx-\frac{\hbar^{2}}{2 m l_{0}^{2}} \frac{\partial^{2}}{\partial x_{\pi}^{2}}+\text { const }
$$

ansieht. Setzt man dann die Eigenfunktionen des Hamilton-Operators des Systems der $\pi$-Elektronen als Slater-Determinante mit (entsprechend den Symmetrieverhältnissen und der Entartung linear kombinierten) Elementen aus (1) an, so erhält man, zumindest für Naphthalin, eine gute Übereinstimmung mit der Erfahrung: Die Absorptionsbanden des Naphthalin erklären, für die Dichte des Systems der $\pi$-Elektronen erhält man, entsprechend der Reaktivität dieses Moleküls, ein Maximum auf den Teilstrecken $\overline{\mathrm{C}_{1} \mathrm{C}_{2}}$, $\mathrm{C}_{3} \mathrm{C}_{4}, \overline{\mathrm{C}_{5} \mathrm{C}_{6}}$ und $\overline{\mathrm{C}_{7} \mathrm{C}_{8}}$. Der erforderliche Aufwand an Rechnungen, der sich mit wachsender Ringzahl noch stark vermehrt, läßt jedoch diesen Lösungsweg als nicht geeignet erscheinen. Im übrigen ist dieses Lösungsverfahren insofern inkonsequent, als die in ihrer Ausbreitungsmöglichkeit auf einzelne $\pi$-Ringe beschränkten Elektronen allein im Feld der $\mathrm{C}^{+}$-Atomrümpfe betrachtet werden, während eine derartige Beschränkung nur im effektiven Molekülfeld, also durch die Mitwirkung der erst nachträglich berücksichtigten Wechselwirkung der $\pi$-Elektronen untereinander, zustande kommen kann.

Meinem verehrten Lehrer, Herrn Professor Dr. KarL BeChert, bin ich für seine Ratschläge und seine Anteilnahme am Fortgang dieser Arbeit dankbar.

15 E. Clar, l. c. ${ }^{3}$, S. 32 . Clar führt die ersten p-Banden der Pyrene auf hypothetische angeregte Formen des Diphenyls zurück.

16 H. Druckrey u. Mitarb., Naturwiss. 39, 17 [1952]. -O. Sснміdт, Z. physik. Chem. 42 (B), 2 [1939]. 